\title{
Statistical Characterization of C-arm Distortion with Application to Intra-Operative Distortion Correction
}

\author{
Gouthami Chintalapani, Ameet K. Jain and Russell H. Taylor \\ Dept. Of Computer Science, The Johns Hopkins University \\ Baltimore, USA
}

\begin{abstract}
C-arm images suffer from pose dependant distortion, which needs to be corrected for intra-operative quantitative $3 \mathrm{D}$ surgical guidance. Several distortion correction techniques have been proposed in the literature, the current state of art using a dense grid pattern rigidly attached to the detector. These methods become cumbersome for intra-operative use, such as 3D reconstruction, since the grid pattern interferes with patient anatomy. The primary contribution of this paper is a framework to statistically analyze the distortion pattern which enables us to study alternate intra-operative distortion correction methods. In particular, we propose a new phantom that uses very few BBs, and yet accurately corrects for distortion.

The high dimensional space of distortion pattern can be effectively characterized by principal component analysis (PCA). The analysis shows that only first three eigen modes are significant and capture about $99 \%$ of the variation. Phantom experiments indicate that distortion map can be recovered up to an average accuracy of less than $0.1 \mathrm{~mm} /$ pixel with these three modes. With this prior statistical knowledge, a subset of BBs can be sufficient to recover the distortion map accurately. Phantom experiments indicate that as few as 15 BBs can recover distortion with average error of $0.17 \mathrm{~mm} /$ pixel, accuracy sufficient for most clinical applications. These BBs can be arranged on the periphery of the C-arm detector, minimizing the interference with patient anatomy and hence allowing the grid to remain attached to the detector permanently. The proposed method is fast, economical, and C-arm independent, potentially boosting the clinical viability of applications such as quantitative 3D fluoroscopic reconstruction.
\end{abstract}

Keywords: distortion, intra-operative distortion correction, principal component analysis, eigen modes

\section{INTRODUCTION}

C-arm fluoroscopy is the most commonly used intra-operative imaging modality because of its low cost and ease of use. These fluoroscopic images are distorted and need to be corrected for use in quantitative intra-operative analysis. This distortion is caused mainly due to the curved nature of the detector resulting in radial or pin cushion type of distortion and due to the earth's magnetic field deflecting the electrons resulting in an S-curve distortion. Boone et al. and Gutierrez et al. discuss the actual physical phenomenon that causes distortion. Moreover, C-arm distortion is pose-dependant, i.e., it varies with the position of the C-arm.

Several C-arm distortion correction techniques have been proposed in literature ${ }^{1-7}$ dating back to 90's. Typically, a dense grid pattern phantom with either metal beads, ${ }^{1,3-6}$ or grooves ${ }^{2}$ is attached rigidly to the image intensifier and is imaged at different C-arm poses. A polynomial, usually a two-dimensional fourth or fifth order polynomial depending on the number of beads in the phantom, is used to model the distortion by mapping the bead coordinates in the fluoroscopic phantom image to the correct geometrical coordinates of the phantom. An interpolation function is then used to define the distortion map for all the pixels in the image. These techniques are robust and are very effective for offline distortion correction.

These grid-based methods become cumbersome for intra-operative use (such as for 3D reconstruction), since the grid pattern either significantly corrupts the patient's image, or necessitates two X-ray shots at each pose (one with and the other without the grid). Fahrig et al. proposes to track C-arm, create a look up table for

Send correspondence to E-mail: greddy@cs.jhu.edu

Medical Imaging 2007: Visualization and Image-Guided Procedures, edited by Kevin R. Cleary, Michael I. Miga, Proc. of SPIE Vol. 6509, 65092Y, (2007) $\cdot 1605-7422 / 07 / \$ 18 \cdot$ doi: 10.1117/12.711605 
a distortion map at each pose and then interpolate the near-by poses to determine the distortion map intraoperatively. This method is fast and overcomes the above mentioned issues, but requires the C-arm to be encoded accurately. The other option would be to remember the poses for which the distortion is measured offline and then repeat the procedure intra-operatively. ${ }^{2}$ This method requires that the C-arm is tracked and the pose is repeatable. Intra-operative $\mathrm{C}$-arm tracking could be slow and expensive.

In this paper, we propose a statistical framework which enables us to analyze the distortion maps at different poses and extract any inherent patterns. We have performed principal component analysis to create a statistical model for distortion patterns exhibited by a C-arm. Although C-arm distortion patterns appear to be random and complicated, our analysis shows that they can be predicted from the principal modes of variation with a very decent accuracy. In particular, we present a novel intra-operative distortion correction application with a phantom which uses very few beads on the periphery of the C-arm image intensifier along with the prior statistical knowledge. This method is fast, economical, and C-arm independent, potentially boosting the clinical viability of applications such as quantitative 3D fluoroscopic reconstruction. The motivation for this work (effective distortion correction) might become obsolete in case of flat panel detector C-arms which would replace existing C-arms in future. However, we believe that this work presents a statistical framework to characterize the distortion patterns effectively and to build applications based on this analysis.

\section{STATISTICAL CHARACTERIZATION OF C-ARM DISTORTION}

\subsection{Method}

We have designed a grid phantom with steel beads, approximately 300 beads, placed at 1 cm distance between beads in a square pattern. Several fluoroscopic phantom images were taken in various C-arm poses by rigidly attaching the phantom to the image intensifier. A basic image processing algorithm is used to segment the bead positions in all the images. These segmented bead positions are referred to as image coordinates $\left(u_{d}, v_{d}\right)$. The actual/nominal bead positions, $\left(u_{o}, v_{o}\right)$, are determined from the physical geometry of the grid phantom and the distortion is modeled using a fifth order Bernstein polynomial as shown in the following equation.

$$
\left(u_{d}, v_{d}\right)=\sum_{i=0}^{n} \sum_{j=0}^{n} C_{i j} B_{i j}\left(u_{0}, v_{0}\right)
$$

where

$$
B_{i j}\left(u_{0}, v_{0}\right)=\left(\begin{array}{c}
n \\
i
\end{array}\right) u_{0}^{i}\left(1-u_{0}\right)^{n-i}\left(\begin{array}{c}
n \\
j
\end{array}\right) v_{0}^{j}\left(1-v_{0}\right)^{n-j}
$$

For each image, a bezier matrix $B$ is constructed using the nominal coordinates of the bead positions and the above equation can be written in matrix form as follows:

$$
\left(U_{o b s}\right)_{m \times 2}=\left(B\left(U_{n o m}\right)\right)_{m \times(n+1)^{2}} \times C_{(n+1)^{2} \times 2}
$$

where $U_{o b s}$ is the array of distorted bead coordinates in the image; $U_{\text {nom }}$ represents the nominal coordinates of the beads; and $m$ being number of grid points on the phantom (here $m=300$ ).

Define a distortion vector as

$$
\triangle \vec{d}=(\triangle u, \triangle v)=\left(u_{d}, v_{d}\right)-\left(u_{0}, v_{0}\right)
$$

The coefficient matrix $C$ can be computed in least squares sense which minimizes the error $\triangle \vec{d}$ for each grid point. This polynomial effectively models the distortion. After computing the polynomial, the distortion vectors for each pixel in the distorted image is computed using 2 and the image is sampled in the resulting distortion map to create a rectified image. In practice, this technique has proved to be robust and accurate with sub-pixel accuracy. 
This procedure is repeated for all the images acquired at different C-arm poses to create distortion maps. In order to extract patterns from these distortion maps, we have performed principal component analysis. The data matrix for this analysis is created by vectorizing the image matrix (distortion map) and by stacking each vector into a matrix $\Delta$ as shown below:

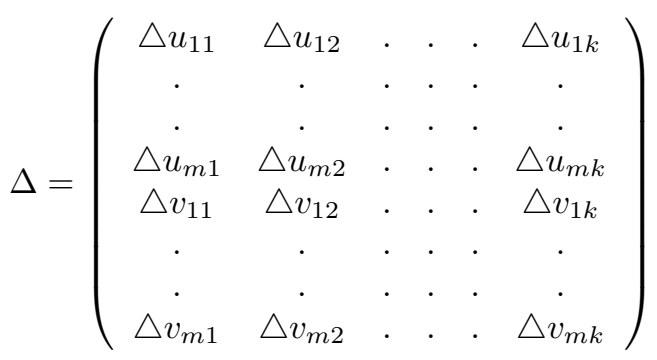

where $\left(\triangle u_{i j}, \Delta v_{i j}\right)$ is the distortion vector of the $i_{t h}$ pixel in the $j_{t h}$ image; $m$ is the number of pixels in the image; and $k$ is the number of images.

For computational issues, we have ignored the background pixels and have only considered the pixels in the active area of the $\mathrm{C}$-arm detector while creating the data matrix. The mean distortion map is computed by taking average of all the distortion maps. This mean value is then substracted from the data matrix to make it a zero mean data matrix, which is essential for principal component analysis. Principal modes of variation (eigen modes) are then extracted from the covariance matrix of the input data matrix. ${ }^{8}$ From this analysis, we can express any distortion map as a linear combination of the principal modes.

$$
\triangle \vec{d}=M_{0}+\sum_{i=1}^{n} \lambda_{i} D_{i}
$$

where $M_{0}$ is the mean distortion map and $D_{i}$ is the $i^{t h}$ principal component. The orthogonal eigen modes represent new basis for the subspace in which the C-arm distortion patterns exist as compared to the very high dimensional parameterization using polynomials. For example, a two dimensional fifth order Bernstein polynomial has 72 coefficients.

\subsection{Results}

We have performed principal component analysis on two sets of distortion maps extracted from the grid phantom images. For the first dataset, we have collected 120 images of the phantom, approximately one every 3 degrees along the propeller axis of an OEC $9600 \mathrm{C}$-arm. We have relied on the gradation marks on the C-arm to position the $\mathrm{C}$-arm and did not use any trackers/encoders. This would be a typical dataset for 3D reconstruction with 2D fluoroscopic images. For the OEC 9600 C-arm, there are two data outputs: 1) DICOM output through floppy and 2)video output through a frame grabber. Typically, floppy output is slow but accurate as compared to the fast but noisy video signal. The image size is 459x484 pixels and 480x720 pixels for floppy and video outputs respectively and the pixel size is 0.44 after distortion correction. We have compared both the dicom and the video images to verify that the video signal does not affect the distortion. All these images were corrected using a fifth order Bernstein polynomial. We then extracted the distortion maps for each image and performed principal component analysis on these distortion maps as explained above.

This analysis shows that the first three principal modes of variation are significant and they capture about 99\% of the variation as shown in Figure 1(a). Figure 1(b) shows the results of leave-out validation test. In this test, of the 120 training images, we have used 60 images, roughly every 6 degrees, to create a statistical model and then fit this model to the remaining 60 images. The error measurement between the true distortion map and the one estimated from the model is given by

$$
\epsilon=\left\|\triangle \vec{d}-\left(M_{0}+\sum_{i=1}^{n} \lambda_{i} D_{i}\right)\right\|^{2}
$$




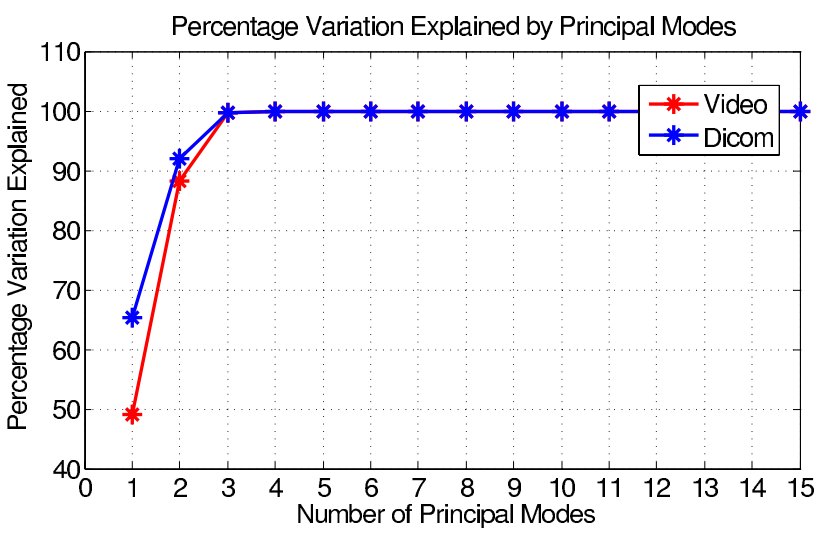

(a) Percentage variance explained by eigen modes

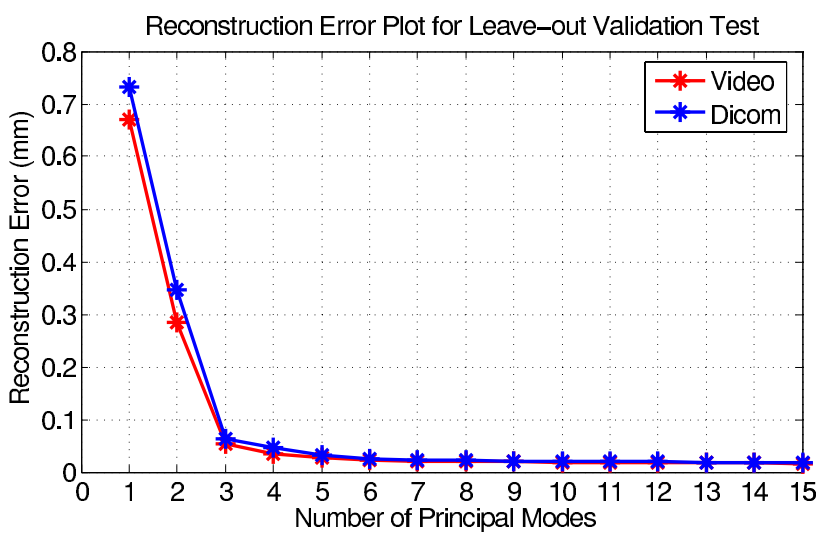

(b) Residual error from leave-out validation test

Figure 1. Principal component analysis on distortion maps. (a) Percentage variation explained by the dominant eigen modes. Note that the first three modes capture about $99 \%$ of the variation. (b) Residual error plot after projecting the leave-out distortion map onto the eigen space as function of number of modes. The average error is less than $1 \mathrm{~mm}$ when the first three principal modes are used. Note that the results from dicom and video images are very similar.

The average raw distortion as measured from the grid phantom is about $2.1488 \mathrm{~mm} /$ pixel with a standard deviation of $1.1469 \mathrm{~mm} /$ pixel and a maximum distortion of $4.6003 \mathrm{~mm} /$ pixel. With the leave-out validation test, we were able to achieve an average distortion correction accuracy of about $0.0632 \mathrm{~mm} /$ pixel and a standard deviation of $0.0294 \mathrm{~mm} /$ pixel with a maximum distortion of $0.202 \mathrm{~mm} /$ pixel using the first three dominant eigen modes. The residual errors for video images were close to the ones from DICOM images and hence validating the fact that either output can be used for statistical analysis. These results indicate that eventhough the Carm distortions are complicated and high-dimensional, they can be represented by the eigen modes and can be predicted fairly accurately.

The actual distortion modes from the principal modes are shown in Figure 2.2. The first principal mode appears similar to barrel distortion, second mode represents S-curve distortion and the third mode looks like a spiral distortion. We have predominantly noticed S-curve distortion in all the images taken with that C-arm. While collecting the data, we have noticed that the images were rotating in one direction until halfway and then in opposite direction for the rest of the sweep. We came to know from the manufacturer that as we move the "C" through it's propeller angles, there is a shift of weight from the bearings on one side of the detector assembly, to the bearing set on the opposite side. This causes a sudden jump and also rotates the images through the sweep. We have included this as part of the distortion model because there is no other way to explicitly measure these inconsistencies and to our surprise these inconsistencies showed up in the mode distribution plots for the mode weight parameters (Figure 2.2). The Figure 3(a) clearly shows the rotational aspect of the C-arm through the propeller axis which is in agreement with the first mode (barrel distortion due to the curvature of the detector). This distortion is more or less assumed to be constant. ${ }^{4,9}$ The rotation caused by the gantry can be clearly seen in this plot. The jump motion can be attributed to the jumps in plots given in Figures 3(b) and 3(c).

For the other experiment, we have collected 200 images distributed more or less uniformly in the sphere spanned by the "C" of the C-arm. We have captured only video images this time as it was convenient to copy images using the frame grabber. Similar eigen analysis on this data says that the first four eigen modes capture $99 \%$ of the variation. The distortion maps defined by the principal modes are shown in Fig 2.2. The first two modes look like barrel distortion along $\mathrm{x}$, y dimensions respectively. The second mode shows the S-curve and the third mode is similar to spiral distortion. From the leave-out validation test, we are able to achieve an average accuracy of $0.07 \mathrm{~mm} /$ pixel, with $0.0553 \mathrm{~mm} /$ pixel standard deviation and $0.3578 \mathrm{~mm} /$ pixel maximum distortion, comparable to the results from the first dataset.

These two datasets are collected with the same phantom on the same C-arm in the same room but at different time periods. The $\mathrm{C}$-arm is displaced with in the room during this time. Qualitatively, the distortion patterns from these two datasets look similar. In order to compare the distortion patterns quantitatively, we measured the 


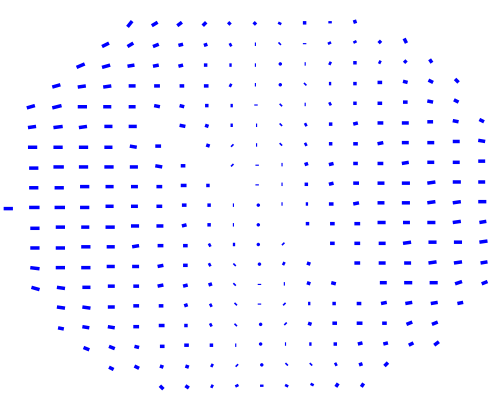

(a) Mean distortion map

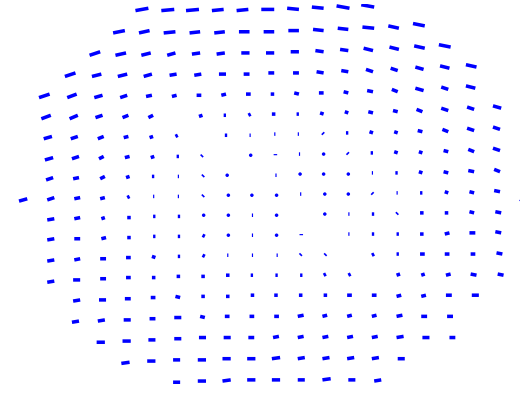

(b) $+3 \sigma_{1}$ Mode 1

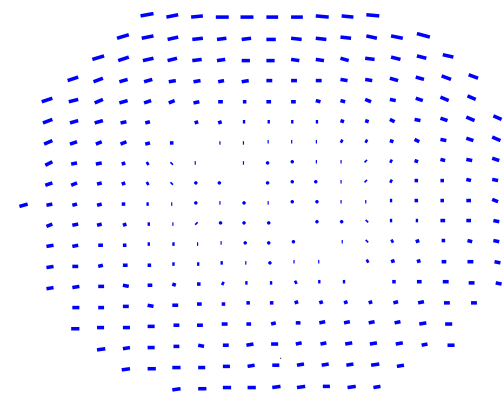

(e) $-3 \sigma_{1}$ Mode 1

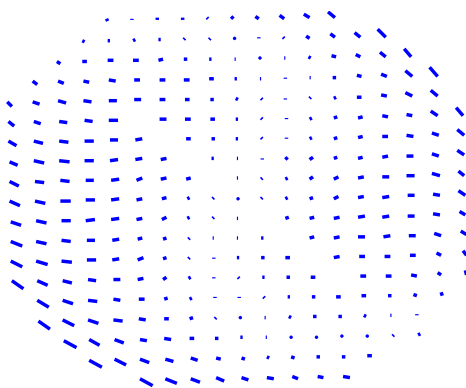

(c) $+3 \sigma_{2}$ Mode 2

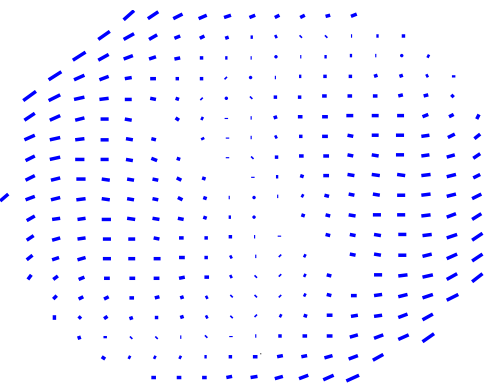

(f) $-3 \sigma_{2}$ Mode 2

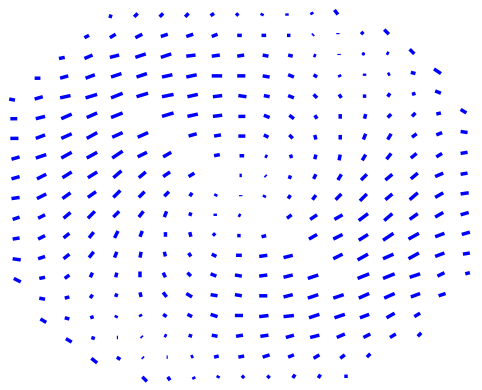

(d) $+3 \sigma_{3}$ Mode 3

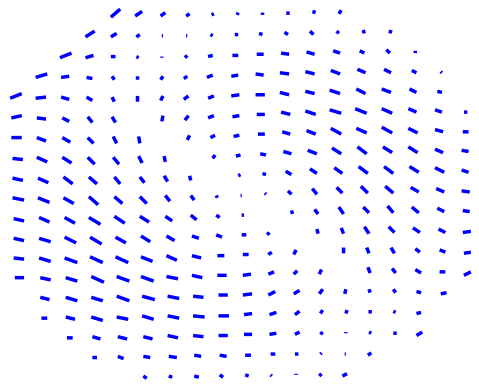

(g) $-3 \sigma_{3}$ Mode 3

Figure 2. Distortion maps as shown by the first three eigen modes from principal component analysis. The first mode is similar to barrel distortion, second mode appears close to an S-curve distortion and the third mode shows spiral distortion

projection errors using equation (4) by projecting one dataset on to the other subspace. This measure quantifies how well one model can generalize the data from the other model. The average distortion correction accuracy is $0.2329 \mathrm{~mm} /$ pixel, with a standard deviation of $0.1157 \mathrm{~mm} / \mathrm{pixel}$ and a maximum distortion correction of 0.6589 $\mathrm{mm} /$ pixel. These results show that the statistical model created from the volume data is capable of modeling the distortion patterns extracted from the full sweep data acquired at a different time. These results strongly indicate that the $\mathrm{C}$-arm distortion patterns are not random and can be predicted with reasonable accuracies. A more controlled experiment with the same C-arm at different locations would provide more insights into this problem.

\section{A NOVEL INTRA-OPERATIVE DISTORTION CORRECTION APPLICATION}

Building upon the statistical framework presented in the previous section, we present a new intra-operative distortion correction method with a phantom that uses very few beads. We propose a new phantom design with beads on the periphery of the image intensifier. These beads can be arranged in any pattern (circular, square etc). The main idea is to use the prior statistical knowledge and the beads to drive the distortion correction process. This design suits intra-operative needs very well because it has very minimal interference with patient 


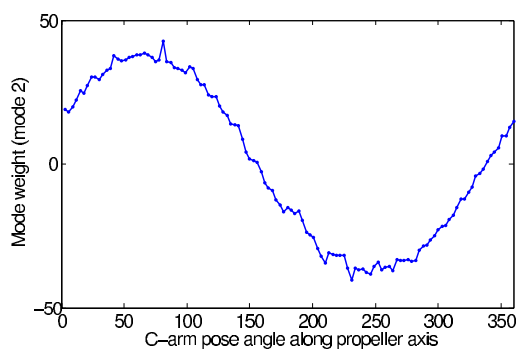

(a) Distributionof $\lambda_{1}$ for 120 images

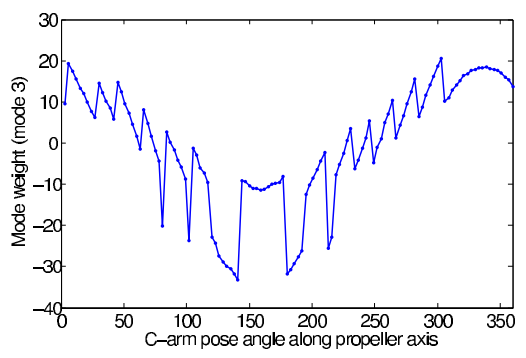

(b) Distributionof $\lambda_{2}$ for 120 images

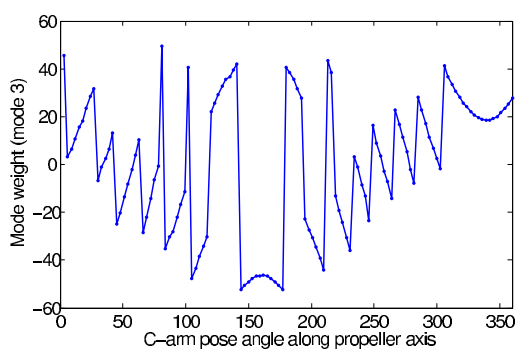

(c) Distributionof $\lambda_{3}$ for 120 images

Figure 3. Distribution of the mode weight parameters for 120 images from a full sweep along the propeller axis

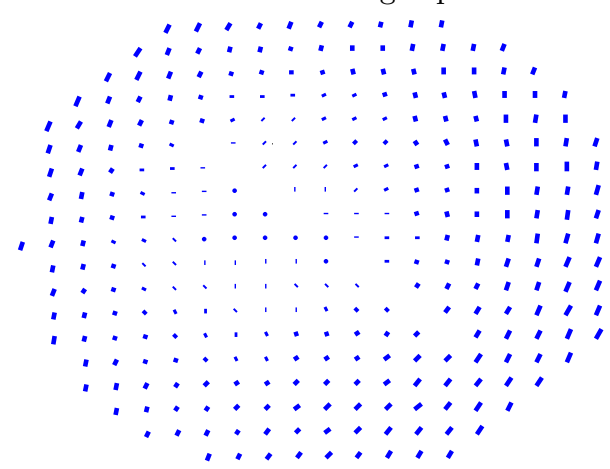

(a) $+3 \sigma_{1}$ Mode 1

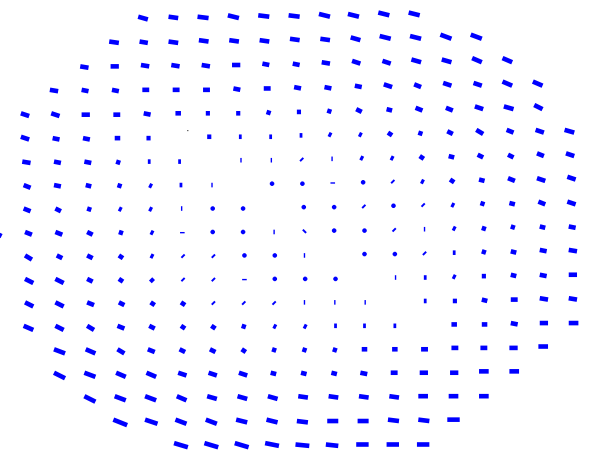

(b) $+3 \sigma_{2}$ Mode 2

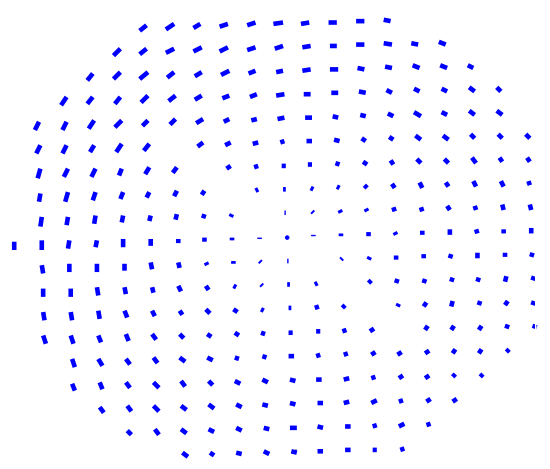

(c) $+3 \sigma_{3}$ Mode 3

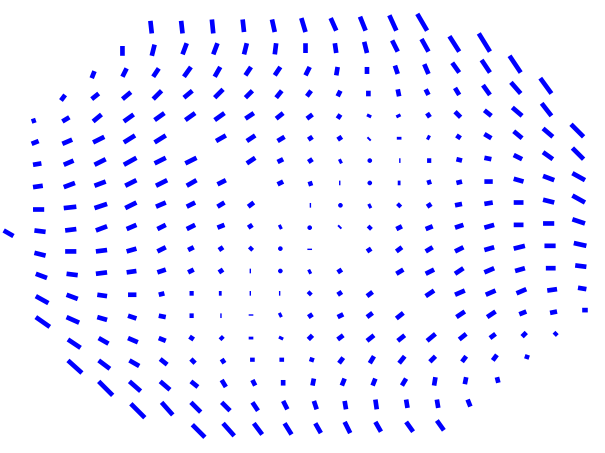

(d) $+3 \sigma_{4}$ Mode 4

Figure 4. Distortion maps as shown by the first four eigen modes from principal component analysis. The first two modes represent barrel distortion in $\mathrm{x}, \mathrm{y}$ directions, second mode shows S-curve type of distortion and the third mode is spiral distortion

anatomy and hence allows the grid to remain attached to the detector through out the surgery. The proposed method is fast, economical, and C-arm independent, potentially boosting the clinical viability of applications such as quantitative 3D fluoroscopic reconstruction. Figure 3 shows our simulation results on the number of beads needed to recover distortion parameters using the prior knowledge. In this simulation, we have used images from the full sweep dataset. Of the 120 images, we have used 60 images to create the mean distortion map and the distortion modes. The remaining 60 images were used to do distortion correction. A band of pixels was selected in each of these 60 images, from which " $n$ " number of pixels were selected as bead locations. These pixels are selected randomly and for each experiment we have performed 100 trials for all the 60 images. For each iteration, we have optimized mode weights by minimizing a similarity measure. The similarity measure is determined by the "squareness"' of the bead locations. By squareness, the bead locations after correction should be on a line and the lines should be orthogonal. In this optimization step, there is no information about 


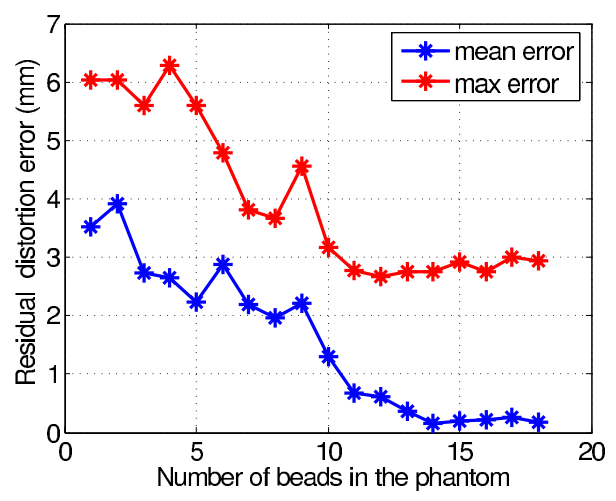

Figure 5. Residual distortion correction error as function of number of beads used for correction

the orientation of the phantom with respect to the image intensifier and hence the bead locations were made to fit a specific pattern, for example a square. We have used downhill simplex optimization method in MATLAB 7.0 with the search space being defined by the distortion modes from PCA. A linear combination of the modes would give a distortion map. This distortion map is interpolated to determine the rectified locations for the beads using bilinear interpolation. The results indicate that as few as 14 or 15 beads are required to recover the distortion parameters with an average accuracy of $0.17 \mathrm{~mm} /$ pixel and a maximum of $2.86 \mathrm{~mm} /$ pixel, accuracy sufficient for most clinical applications. The maximum error is high because the objective function is not able to capture the distortions. A slight modification to the objective function incorporating the orientation of the phantom might improve the results significantly.
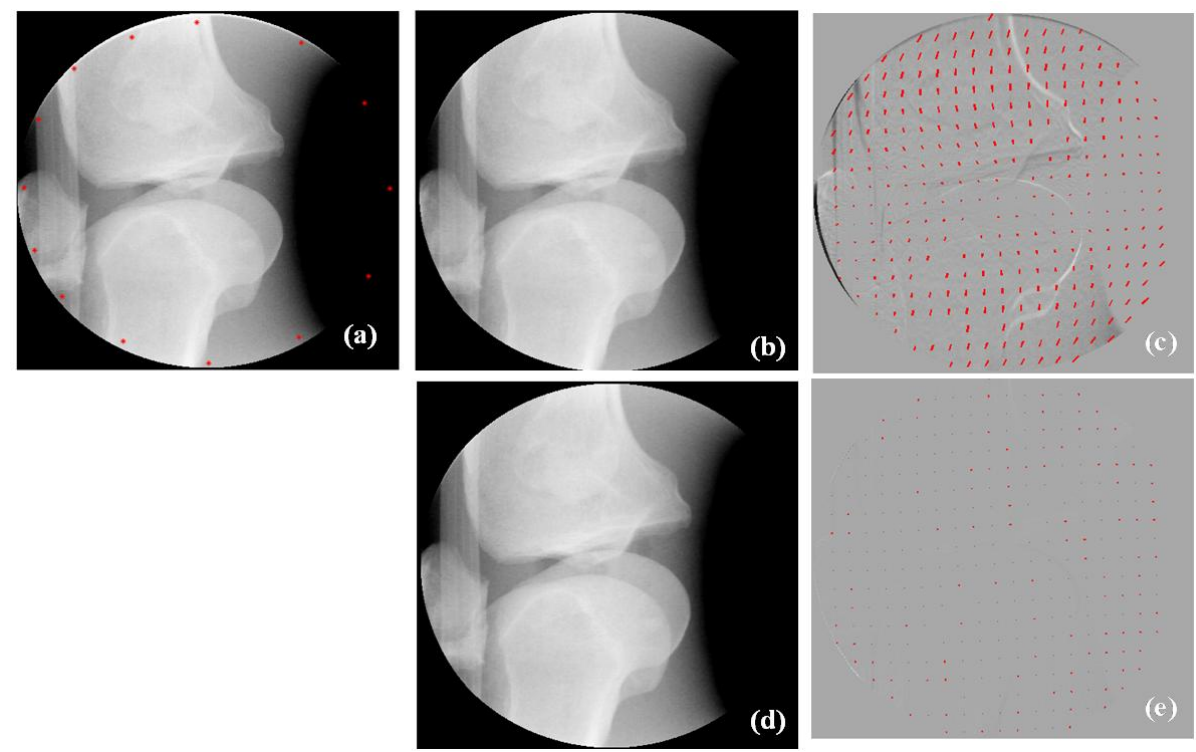

Figure 6. Simulation of the new phantom design. (a) shows the fluoroscopic image of a knee with the phantom beads highlighted in red (b) distortion corrected image using the dense grid phantom (c) true distortion map overlaid on difference image ((a)- (b)). (d) distortion corrected image using the beads from (a) and statistical modes (e) residual distortion map after correction using new phantom overlaid on the gray scale difference image ( (c)-(d)) 


\section{CONCLUSIONS AND FUTURE WORK}

In this paper, we have presented a statistical framework to analyze C-arm distortion patterns. Principal component analysis on distortion maps at different orientations of the $\mathrm{C}$-arm reveal the inherent patterns. Our results from two datasets indicate that even though the distortion patterns are high dimensional and complicated, they can be predicted fairly accurately using the first three to four principal modes. This analysis can be easily extended to study the distortion patterns over time, and at different locations and across different C-arms, adding more dimensions to the problem. Such analysis would give better insights in to modeling distortion patterns effectively. Apparently, this work is not applicable to flat panel detector C-arms. Nevertheless, any one using non-flat-panels have to go through the tedious distortion correction procedure. In such cases, we consider that this statistical approach would address most of the current issues with the C-arm distortion correction.

We have described a novel intra-operative distortion correction method that uses a phantom with very few beads. Our simulation experiments have shown that as few as 15 beads can recover the distortion parameters with an average accuracy of $0.17 \mathrm{~mm} /$ pixel. This new phantom has very minimal interference with the patient anatomy and hence can remain attached to the C-arm during surgery. We believe that this method is fast and practical and can be used effectively for intra-operative applications such as 3D reconstruction. Moving further away from the phantoms, another approach would be to do a phantom less distortion correction of patient images using patient $\mathrm{CT}$ as a fiducial. The $2 \mathrm{D}$ views from $3 \mathrm{D}$ patient $\mathrm{CT}$ can be compared to the actual x-ray images of the patient to optimize the distortion modes, hence catering to the needs of intra-operative applications.

\section{ACKNOWLEDGMENTS}

This work is supported in part by NSF ERC Grant EEC9731478, by NIH/NIBIB research grant R21-EB003616 and by DOD PC050170. We would also like to thank Iulian Iordachita for making the calibration phantom and Gabor Fichtinger for his assistance in this project.

\section{REFERENCES}

1. R. Fahrig, M. Moreau, and D.W. Holdsworth, "Three-dimensional computed tomographic reconstruction using a c-arm mounted xrii: correction of image intensifier distortion," Med Phys, vol. 24(7), pp. 1097-106, 1997.

2. J. Yao, R.H. Taylor, R.P. Goldberg, R. Kumar, A. Bzostek, R.V. Vorhis, P. Kazanzides, and A. Gueziec, "Robust automatic c-arm calibration for fluoroscopy-based navigation: A practical approach," in Computer Aided Surgery (CAS), 2000, vol. 5(6), pp. 373-90.

3. H. Livyatan, Z. Yaniv, and L. Joskowicz, "Robust automatic c-arm calibration for fluoroscopy-based navigation: A practical approach," in MICCAI, 2002, vol. 2489, pp. 60-68.

4. L. F. Gutierrez, G. Shechter, R. J. Lederman, E.R. McVeigh, and C. Ozturk, "Distortion correction, calibration, and registration: Towards an integrated $\mathrm{mr}$ and x-ray interventional suite," in Proc. of SPIE, Medical Imaging 2005: Visualization, Image-Guided Procedures, and Display, 2005, vol. 5744, pp. 146-156.

5. S. Schreiner, J.H. Anderson, R.H. Taylor, J. Funda, A.Bzostek, and A.C. Barnes, "A system for percutaneous delivery of treatment with a fluoroscopically-guided robot," in In Troccaz J, Grimson E, Mosges R, editors: Proceedings of First Joint Conference on Computer Vision, Virtual Reality and Robotics in Medicine and Medical Robotics and Computer Assisted Surgery (CVRMed-MRCAS' 97), Grenoble, France, March 1997, 1997, pp. 747-756.

6. R. Hofstetter, M. Slomczykowski, M. Sati, and L. Nolte, "Fluoroscopy as an imaging means for computerassisted surgical navigation," in Proc. of Computer Aided Surgery (CAS), 1999, vol. 4(2), pp. 65-76.

7. B. Schueler and X. Hu, "Correction for image intensifier distortion for three-dimensional x-ray angiography," in Proc. of SPIE, Medical Imaging 1995, 1995, vol. 2432, pp. 272-279.

8. I.T. Jolliffe, "Principal component analysis," Berlin: Springer Verlag, 1986.

9. Boone J.M., Seibert J.A., Barrett W.A., and Blood E.A., "Analysis and correction of imperfections in the image intensifier-tv-digitizer imaging chain," Med Phys, vol. 18, pp. 236-242, 1991. 